\title{
Solid waste management transformation and future challenges of source separation and recycling practice in Malaysia
}

\begin{abstract}
The mentality towards cleanliness, the sense of responsibility towards properly managing waste, as well as public concerns on the implications of not separating waste for recycling are critically lacking. Besides widespread of open dumping and illegal dumping, landfill sites in Malaysia are in dire state while source separation for recycling remain minimal despite the dominance of recyclable materials in the waste composition. The historical discussion on the solid waste management policy and plan strategies assesses Malaysian solid waste management needs to set realistic perspective for solid waste management particularly in source separation and recycling. Transformation of primitive solid waste management policy and plan strategies resulted to major changes in the system and rigorous implementation of mandatory source separation through Act 672. A two-pronged strategy of federalization and privatization is formally implemented in eight states of Peninsular Malaysia, Federal Territory of Kuala Lumpur, and Putrajaya. In line with the government's effort to promote sustainable solid waste management services, SWCorp Malaysia implemented SWCorp Strategic Plan 2014-2020, which focuses on (1) mindset, (2) behavior and culture, (3) collaboration and synergy, (4) policy and regulations, (5) organizational capacity, (6) technology system and facilities, (7) law enforcement, and (8) delivery system. One of the most critical challenges in source separation and recycling practice is the public attitude towards making source separation and recycling as a habit. Continuous commitment and participation from the government, private sector, and public are essential to achieve Malaysia's targeted recycling rate of $22 \%$ by 2020 , with greater advancement towards a zero waste nation.
\end{abstract}

Keyword: Source separation; Recycling; Solid waste management; Policy implementation; Plan strategies; Malaysia 
